\title{
Estrogen and progesterone receptor expression levels do not differ between lobular and ductal carcinoma in patients with hormone receptor-positive tumors
}

\author{
Wilfred Truin $^{1}$ - Rudi M. H. Roumen ${ }^{1,2}$ - Sabine Siesling ${ }^{3,4} \cdot$ Koen K. van de Vijver ${ }^{5}$. \\ Vivianne C. G. Tjan-Heijnen ${ }^{2} \cdot$ Adri C. $\operatorname{Voogd}^{2,3,6}$
}

Received: 22 March 2017 / Accepted: 23 March 2017/Published online: 1 April 2017

(c) The Author(s) 2017. This article is an open access publication

\begin{abstract}
Background Differences in estrogen (ER) and progesterone (PR) expression between invasive lobular carcinoma (ILC) and invasive ductal carcinoma (IDC) could be an underlying reason for the difference in chemo-sensitivity and response to hormonal therapy between ILC and IDC. The aim of this study was to investigate the differences in ER and PR expression levels between postmenopausal patients with hormonal receptor-positive ILC and IDC.

Methods We included all ER and/or PR receptor-positive ILC and IDC, diagnosed between January 2011 and December 2013 from the population-based Netherlands Cancer Registry. A semi-quantitative classification was used to analyze differences in ER/PR expression, which consisted of three ER expression classes: 10-69, 70-89, and $\geq 90 \%$. Differences in ER and PR expression levels between IDC and ILC were analyzed according to age
\end{abstract}

Adri C. Voogd

adri.voogd@maastrichtuniversity.nl

1 Department of Surgery, Máxima Medical Center, Veldhoven, The Netherlands

2 Department of Medical Oncology, GROW - School for Oncology and Developmental Biology, Maastricht University Medical Center, Maastricht, The Netherlands

3 Department of Research, Netherlands Comprehensive Cancer Organisation, Utrecht, The Netherlands

4 Department of Health Technology and Services Research, MIRA Institute for Biomedical Technology and Technical Medicine, University of Twente, Enschede, The Netherlands

5 Department of Pathology, The Netherlands Cancer Institute, Amsterdam, The Netherlands

6 Department of Epidemiology, Maastricht University Medical Center, PO Box 616, 6200 MD Maastricht, The Netherlands group, tumor size, axillary nodal status, grade, and HER2 status.

Results In total, 26,339 ER and/or PR-positive breast cancers were included in the study, of which $17 \%$ were ILC and $83 \%$ IDC. In patients with IDC, $86 \%$ of the tumors showed an ER expression level of $90 \%$ or more, compared to $84 \%$ in those with ILC. In both IDC and ILC a PR expression level of $90 \%$ or more was observed in $54 \%$ of the tumors. In postmenopausal patients aged 50-69 years no significant differences could be observed in ER and PR expression levels between ILC and IDC.

Conclusion Patients with ER and PR-positive ILC and IDC have similar quantitative ER and PR expression profiles, implicating that ER/PR expression is unlikely to be a confounding factor in studies concerning chemo-sensitivity of ILC and IDC.

Keywords Invasive lobular carcinoma - Invasive ductal carcinoma $\cdot$ Estrogen receptor $\cdot$ Progesterone receptor

\section{Introduction}

Next to invasive ductal carcinoma (IDC), invasive lobular carcinoma (ILC) is the second most common type of breast cancer, representing approximately $15 \%$ of all breast tumors. ILC is unique in its biological and clinical behavior, with a lower E-cadherin expression and a greater likelihood of being hormone receptor positive than IDC. Local control and survival is reported to be similar in patients with ILC and IDC [1,2].

In modern treatment guidelines, no discrimination is made between ILC and IDC regarding use of systemic treatment [3], even though previous studies have shown an inferior response of estrogen (ER) and/or progesterone 
(PR)-positive ILC to neoadjuvant chemotherapy, compared to ER/PR-positive IDC. This difference is most pronounced when looking at the rates of pathological complete response. Reported proportions of patients with a pathological complete response range from 0 to $5 \%$ for patients with ER/PR-positive ILC, compared to 6 to $20 \%$ for those with ER/PR-positive IDC [4-6]. In contrast, significantly higher rates of pathological complete response, ranging up to almost $18 \%$, are seen in the small portion of patients with ER/PR negative and poorly differentiated ILC, suggesting an important role for hormonal receptor status in this histological subgroup [7]. Furthermore, studies from our group showed that adjuvant chemotherapy seems to confer no additional beneficial effect to hormonal therapy in postmenopausal patients with primary non-metastatic ILC. In contrast, patients with IDC showed a relative risk reduction in mortality of about $17 \%$ with the addition of chemotherapy to hormonal treatment in the adjuvant setting $[8,9]$.

An important limitation in studies comparing the effect of adjuvant treatment in ILC and IDC is the lack of data concerning quantitative ER and PR levels, which could possibly be a confounding factor when interpreting these results. Quantitative data about the ER/PR expression in these patients could elucidate the question whether it is a higher ER/PR level or another intracellular signaling pathway related to histology that explains the lower chemo-sensitivity or higher response to endocrine therapy in ILC, compared to IDC. Therefore, we questioned if a difference exists in the quantitative ER and PR status of patients with ILC or IDC.

\section{Methods}

\section{Patients}

All female patients with ER and/or PR receptor-positive ILC or IDC diagnosed between January 2011 and December 2013 were selected from the population-based Netherlands Cancer Registry. According to Dutch guidelines, breast tumors were called ER or PR positive when the expression level was $10 \%$ or higher.

The registry records data on all patients with a new diagnosis of in situ and invasive tumors in the Netherlands. Trained registry managers prospectively collected data from medical records after notification, which are mainly obtained from the automated pathology archive (PALGA). Other sources used were the National Registry of Hospital Discharge Diagnoses and the databases of the radiotherapy departments. Data about patient, tumor, and treatment characteristics were collected from patient hospital files.
In total, 46,022 invasive breast cancers were diagnosed in the years 2011-2013. From these 46,022 breast cancers, we excluded 2271 tumors who were diagnosed together with distant metastatic disease and 3225 tumors who were not treated with surgery. From the remaining 40,526 breast cancers we excluded those for which neoadjuvant chemotherapy was used $(n=4789)$ and selected only breast cancers of either lobular or ductal histology, leaving a total 33,441 breast cancers.

\section{Statistical analyses}

For each breast tumor, the percentage of ER and PR expression was derived from the database. The primary study endpoint of the study is the distribution of ER and PR expression levels in patients with hormone receptor-positive ILC, as compared with hormone receptor-positive IDC. To analyze differences in ER and PR expression levels between ILC and IDC, a semi-quantitative classification was used, which consisted of three ER expression classes: 10-69, 70-89 and >90\%. These analyses were stratified according to age group, postoperative nodal status, postoperative tumor size, tumor grade, and HER2 status. Differences in patient characteristics between patients with IDC or ILC were calculated using the $\chi^{2}$ test.

\section{Results}

\section{Characteristics}

ER or PR status was missing for 509 (1.5\%) of the 33,441 tumors selected for the study. A positive ER status was observed in 26,118 tumors $(78,1 \%)$, and a positive PR status in 21,348 tumors $(63,8 \%)$. In 6645 tumors $(10.9 \%)$, the ER and the PR status were both negative, and in 21,179 tumors $(63.3 \%)$ both receptors were positive. In total, 26,339 ER or PR-positive breast cancers were included in the study, of which $17 \%$ were ILC and $83 \%$ IDC. Characteristics of these tumors are shown in Table 1. Age at diagnosis of patients with ILC was somewhat higher than age at diagnosis of those with IDC. Although IDCs were smaller, they were more likely to be poorly differentiated (grade 3 ), compared to ILCs. IDCs were more often HER2-positive, compared to ILCs.

\section{ER expression in ILC and IDC}

The distribution of the ER expression level in ILC and IDC is presented in Fig. 1, and the expression levels in different subgroups is shown in Table 2. In patients aged 50-69 years, the proportion of patients with a high ER expression level $(\geq 90 \%)$ was somewhat smaller in those with ILC than in those with IDC (84 vs. $87 \%$, respectively). 
Table 1 General characteristics of estrogen receptor or progesterone receptor-positive invasive lobular (ILC) or invasive ductal (IDC) breast cancers, diagnosed between 2011-2013

\begin{tabular}{|c|c|c|c|c|c|}
\hline \multirow[t]{2}{*}{ Characteristic } & \multicolumn{2}{|c|}{$\operatorname{ILC}(n=4513)$} & \multicolumn{2}{|c|}{ IDC $(n=21,826)$} & \multirow[t]{2}{*}{$P$} \\
\hline & No. & $\%$ & No. & $\%$ & \\
\hline \multicolumn{6}{|c|}{ Age at diagnosis (years) } \\
\hline$<50$ & 675 & (15) & 4050 & (19) & \multirow[t]{3}{*}{$<0.0001$} \\
\hline $50-69$ & 2512 & (56) & 12,348 & $(57)$ & \\
\hline$\geq 70$ & 1326 & (29) & 5428 & $(25)$ & \\
\hline \multicolumn{6}{|c|}{ Tumor size $(\mathrm{cm})$} \\
\hline$<1$ & 691 & (15) & 5180 & (23) & \multirow[t]{4}{*}{$<0.0001$} \\
\hline $1-2$ & 1710 & (38) & 10,438 & $(48)$ & \\
\hline$>2$ & 2049 & (45) & 6006 & $(28)$ & \\
\hline Unknown & 63 & (1) & 202 & (1) & \\
\hline \multicolumn{6}{|c|}{ Axillary nodal status } \\
\hline Negative & 2840 & (63) & 14,418 & (66) & \multirow[t]{3}{*}{0.0002} \\
\hline Positive & 1600 & (35) & 7056 & (32) & \\
\hline Unknown & 73 & (2) & 352 & (2) & \\
\hline \multicolumn{6}{|l|}{ Grade } \\
\hline 1 & 773 & (17) & 6364 & (29) & \multirow[t]{4}{*}{$<0.0001$} \\
\hline 2 & 3127 & (69) & 9883 & $(45)$ & \\
\hline 3 & 416 & (9) & 4970 & (23) & \\
\hline Unknown & 197 & (4) & 609 & (3) & \\
\hline \multicolumn{6}{|l|}{ HER2 status } \\
\hline Negative & 4255 & (94) & 19,229 & $(88)$ & \multirow[t]{3}{*}{$<0.0001$} \\
\hline Positive & 184 & (4) & 2255 & (10) & \\
\hline Unknown & 74 & (2) & 342 & (2) & \\
\hline
\end{tabular}

Also in patients aged 70 years or older, the group with high ER expression levels was somewhat smaller in those with ILC, compared to those with IDC (88 vs. 91\%, respectively). In patients with small tumors, well-differentiated tumors, HER2-positive tumors, or a negative axillary lymph node status, the ER expression level was higher in IDC than in ILC. The ER expression level was higher in patients with ILC than in patients with IDC for those with larger tumors and poorly differentiated tumors.

\section{PR expression in ILC and IDC}

The distribution of the PR expression level in ILC and IDC is presented in Fig. 2, and Table 3 shows the PR expression levels in different subgroups. In patients with ILC, the proportion with a high PR expression level $(\geq 90 \%)$ was $54 \%$, compared to a similar $54 \%$ in those with IDC. In patients aged $<50$ years, the proportion of patients with a high PR expression level ( $\geq 90 \%)$ was somewhat higher in those with ILC than in those with IDC (63 vs. 58\%, respectively). In patients aged 70 years or older, the group with a high PR level was smaller in those with ILC, compared to those with IDC (49 vs. $54 \%$, respectively). No remarkable differences in PR levels were observed between ILC and IDC in stratified analyses according to tumor size and axillary nodal status. The PR expression level was higher in poorly differentiated ILC, compared to poorly differentiated IDC (51 vs. $41 \%$, respectively).

\section{Discussion}

In the present study, performed with prospectively collected data from the Netherlands Cancer Registry, statistically significant differences in ER and PR expression levels were observed between patients with ILC and IDC. However, the absolute differences were very small and are unlikely to be of clinical relevance. In ER-positive breast cancer patients, more than $80 \%$ showed an expression level of more than $90 \%$. Especially in postmenopausal patients aged 50-69 years with ER-positive breast cancer, no significant differences could be observed in ER expression levels between ILC and IDC patients. Furthermore, the differences in PR expression levels between ILC and IDC were even smaller, compared to ER levels. We are not aware of previous studies reporting on highly detailed ER/ PR expression levels of ILC and IDC in a large dataset of more than 25,000 patients.

The primary reason to perform the present study was to determine if differences in quantitative ER/PR expression between ILC and IDC could be an important limitation for the interpretation of the results of our previously published study [9]. In that study, we found that adding chemotherapy to hormonal treatment did not improve the overall survival of patients with ILC, in contrast to those with IDC, who clearly benefited from the use of chemotherapy. According to criticasters, this result could partially be explained by the better response of ILC to hormonal therapy due to higher ER expression levels, compared to patients with IDC [10]. In the present study, we proved that among patients with ER-positive breast cancer, there were no clinically relevant differences with respect to ER/PR expression levels. This conclusion suggests that it is the lobular histology itself that relates to the lower chemosensitivity compared to the ductal counterpart.

The discussion about the reduced chemo-sensitivity of ILC is ongoing for several years now. The added value of (neo)adjuvant chemotherapy in ER-positive ILC was already questioned in reviews by Katz et al. and Purushotham et al. [11, 12]. In several randomized neoadjuvant trials and population-based case series, patients with ILC were shown to have a significantly lower pathological complete response percentages, compared to patients with IDC [4-6]. Also in a recent, pooled analysis of nine neoadjuvant trials including 1052 patients with ILC, a low percentage of 
Table 2 Percentage of estrogen receptor (ER) expression in ER-positive invasive lobular (ILC) or invasive ductal (IDC) breast cancer

\begin{tabular}{|c|c|c|c|c|c|c|c|}
\hline \multirow[t]{2}{*}{ Characteristic } & \multicolumn{3}{|c|}{ ILC $(n=4498)$ ER expression group (\%) } & \multicolumn{3}{|c|}{ IDC $(n=21,667)$ ER expression group $(\%)$} & \multirow[t]{2}{*}{$P$ value } \\
\hline & $10-69 \%$ & $70-89 \%$ & $\geq 90 \%$ & $10-69 \%$ & $70-89 \%$ & $\geq 90 \%$ & \\
\hline \multicolumn{8}{|c|}{ Age at diagnosis (years) } \\
\hline$<50$ & $51(8)$ & $113(17)$ & $507(75)$ & $443(11)$ & $616(15)$ & $2952(74)$ & 0.0227 \\
\hline $50-69$ & $121(5)$ & $268(11)$ & $2116(84)$ & $571(5)$ & $991(8)$ & $10,705(87)$ & $<.0001$ \\
\hline$\geq 70$ & $39(3)$ & $121(9)$ & $1162(88)$ & $184(3)$ & $320(6)$ & $4887(91)$ & 0.0001 \\
\hline \multicolumn{8}{|c|}{ Tumor size $(\mathrm{cm})$} \\
\hline$<1$ & $26(4)$ & $83(12)$ & $578(84)$ & $221(4)$ & $393(8)$ & $4537(88)$ & 0.0003 \\
\hline $1-2$ & $81(5)$ & $169(10)$ & $1456(85)$ & $521(5)$ & $880(8)$ & $8975(87)$ & 0.144 \\
\hline$>2$ & $99(5)$ & $247(12)$ & $1696(83)$ & $445(8)$ & $637(11)$ & $4858(81)$ & $<.0001$ \\
\hline \multicolumn{8}{|c|}{ Axillary nodal status } \\
\hline Negative & $132(5)$ & $301(11)$ & $2401(84)$ & $709(5)$ & $1168(8)$ & $12,444(87)$ & $<.0001$ \\
\hline Positive & $76(5)$ & $195(12)$ & $1321(83)$ & $480(7)$ & $738(10)$ & $5783(83)$ & 0.002 \\
\hline \multicolumn{8}{|l|}{ Grade } \\
\hline 1 & $33(4)$ & $73(9)$ & $665(87)$ & $170(3)$ & $439(7)$ & $5732(90)$ & 0.001 \\
\hline 2 & $145(5)$ & $338(11)$ & $2633(84)$ & $418(4)$ & $828(8)$ & $8597(88)$ & $<.0001$ \\
\hline 3 & $21(5)$ & $57(14)$ & $336(81)$ & $581(12)$ & $613(13)$ & $3689(75)$ & 0.0001 \\
\hline \multicolumn{8}{|l|}{ HER2 status } \\
\hline Negative & $183(4)$ & $469(11)$ & $3589(85)$ & 798 (4) & $1532(8)$ & $16,774(88)$ & $<.0001$ \\
\hline Positive & $24(13)$ & $28(15)$ & $131(72)$ & $392(17)$ & $378(17)$ & $1457(66)$ & 0.199 \\
\hline
\end{tabular}

Table 3 Percentage of progesterone receptor (PR) expression in PR-positive invasive lobular (ILC) or invasive ductal (IDC) breast cancer

\begin{tabular}{|c|c|c|c|c|c|c|c|}
\hline \multirow[t]{2}{*}{ Characteristic } & \multicolumn{3}{|c|}{ ILC $(n=3561)$ PR expression group (\%) } & \multicolumn{3}{|c|}{ IDC $(n=17,790)$ PR expression group $(\%)$} & \multirow[t]{2}{*}{$P$ value } \\
\hline & $10-69 \%$ & $70-89 \%$ & $\geq 90 \%$ & $10-69 \%$ & $70-89 \%$ & $\geq 90 \%$ & \\
\hline \multicolumn{8}{|c|}{ Age at diagnosis (years) } \\
\hline$<50$ & $117(18)$ & $122(19)$ & $407(63)$ & $823(23)$ & $681(19)$ & $2041(58)$ & 0.010 \\
\hline $50-69$ & $615(32)$ & $339(17)$ & $989(51)$ & $3079(31)$ & $1816(18)$ & $4978(50)$ & 0.611 \\
\hline$\geq 70$ & $318(33)$ & $180(19)$ & $474(49)$ & $1268(29)$ & $761(17)$ & $2343(54)$ & 0.021 \\
\hline \multicolumn{8}{|c|}{ Tumor size $(\mathrm{cm})$} \\
\hline$<1$ & $136(26)$ & $96(18)$ & $296(56)$ & $1164(27)$ & $740(17)$ & $2335(55)$ & 0.698 \\
\hline $1-2$ & $390(28)$ & $248(18)$ & $737(54)$ & $2371(27)$ & $1600(19)$ & $4666(54)$ & 0.759 \\
\hline$>2$ & $508(32)$ & $286(18)$ & $817(51)$ & $1596(34)$ & 896 (19) & $2270(48)$ & 0.107 \\
\hline \multicolumn{8}{|c|}{ Axillary nodal status } \\
\hline Negative & $641(29)$ & $402(18)$ & $1171(53)$ & 3265 (28) & 2125 (18) & $6375(54)$ & 0.461 \\
\hline Positive & $391(30)$ & $234(18)$ & $668(52)$ & $1831(32)$ & 1075 (19) & $2836(49)$ & 0.329 \\
\hline \multicolumn{8}{|l|}{ Grade } \\
\hline 1 & $168(28)$ & $107(18)$ & $332(55)$ & $1295(24)$ & $975(18)$ & $3216(59)$ & 0.073 \\
\hline 2 & $729(30)$ & $439(18)$ & $1300(53)$ & $2306(28)$ & $1496(18)$ & $4369(53)$ & 0.436 \\
\hline 3 & $108(32)$ & $56(17)$ & $172(51)$ & 1423 (39) & $713(20)$ & $1510(41)$ & 0.002 \\
\hline \multicolumn{8}{|l|}{ HER2 status } \\
\hline Negative & $984(29)$ & $610(18)$ & $1790(53)$ & $4390(27)$ & 2933 (18) & 8677 (54) & 0.151 \\
\hline Positive & $47(38)$ & $19(15)$ & $57(46)$ & $702(46)$ & 288 (19) & $524(35)$ & 0.033 \\
\hline
\end{tabular}

pathologic complete response was observed. However, the lower response rate did not translate into a poorer long-term outcome. Based on the lower response rates, it was recommended in this study that the use of neoadjuvant chemotherapy in patients with ILC should be restricted to the small proportion with ER-negative disease [7]. Altogether, these data contribute to the assumption that ER-positive ILC and IDC respond differently to chemotherapy. 
Fig. 1 Percentage of estrogen receptor (ER) expression in ERpositive invasive lobular (ILC) or invasive ductal (IDC) breast cancer

Fig. 2 Percentage of progesterone receptor $(\mathrm{PR})$ expression in PR-positive invasive lobular (ILC) or invasive ductal (IDC) breast cancer
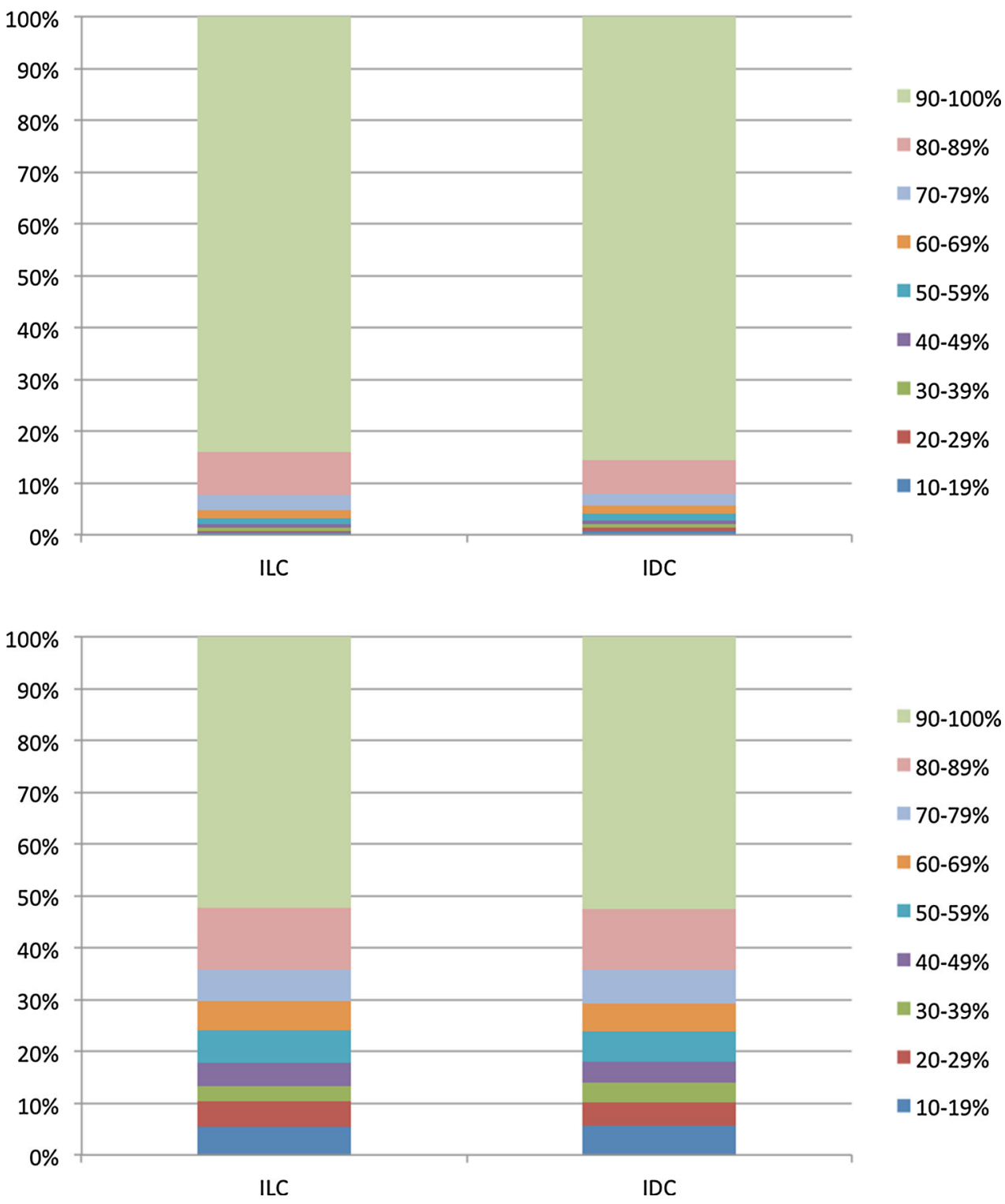

Concerning the response of ILC and IDC to adjuvant hormonal treatment of breast cancer, a study by Rakha et al. showed that the response was better in patients with ILC and that they had a better survival as compared to matched patients with IDC [13]. A more recent study by van de Water et al. showed a similar effect of endocrine therapy regimens in IDC and ILC. On the other hand, they also reported that patients with ER-rich tumors experienced a larger benefit of upfront Exemestane, while patients with ER-poor tumors had better outcomes with sequential therapy, irrespective of histological subtype [14]. This finding emphasizes the relevance of quantification of ER expression levels in hormonal treatment strategies. In concordance with our study, van de Water et al. observed no significant differences between ILC and IDC when looking at semi-quantitative ER expression levels.
Furthermore, studies investigating progesterone as a predictive marker for response to endocrine therapy show that loss of PR expression predicts relative resistance to tamoxifen, whereas maintenance of response to aromatase inhibitors can be observed, suggesting a selective role of this treatment in this subgroup [15-17].

In the current era of molecular characterization of breast cancer, most patients with ILC should be classified as luminal A, since ILC is high in ER and PR expression, often low grade and most often HER2-negative [16]. In general, luminal A type tumors do show a good responsiveness to hormonal therapy and because of this, tamoxifen and aromatase inhibitors serve as keystone therapies in ER/PR-positive ILC. This makes it difficult to prove if the good prognosis of patients with lobular breast cancer treated with endocrine therapy, and the apparent lack of an 
additional effect of chemotherapy, is the result of an excellent response to endocrine treatment or of a reduced chemo-sensitivity. In this light, the recent St Gallen guideline also stated that it is among these patients with the 'luminal' type of breast cancer, of which ILC is typical example, that uncertainty exists whether to use adjuvant chemotherapy [3].

Some limitations in this study should be considered when interpreting its results. Despite the fact that these data are derived from a large prospectively collected dataset, some missing values were observed with respect to tumor size, axillary nodal status, grade, HER2 status, and ER expression. However, the number of missing values is too small to have a real and relevant impact on the results. Moreover, information on other tumor characteristics, such as lymphovascular invasion was not available in this database.

In conclusion, our study provides strong evidence that, when looking at patients with ER and PR-positive breast cancer, ILC and IDC do not differ with respect to quantitative ER and PR expression levels. This finding provides additional proof for the lower chemo-sensitivity of ILC and the opinion that histological subtype should play an important role in the decision-making process regarding the use of chemotherapy in this patient subgroup. Future (neo)adjuvant randomized studies or analyses of existing trial data are warranted to provide further evidence on this subject.

\section{Compliance with ethical standards}

Conflict of interest The authors declare that there is no conflict of interest that could be perceived as prejudicing the impartiality of the research reported.

Funding This research did not receive any specific grant from any funding agency in the public, commercial, or not-for-profit sector.

Open Access This article is distributed under the terms of the Creative Commons Attribution 4.0 International License (http://crea tivecommons.org/licenses/by/4.0/), which permits unrestricted use, distribution, and reproduction in any medium, provided you give appropriate credit to the original author(s) and the source, provide a link to the Creative Commons license, and indicate if changes were made.

\section{References}

1. Fortunato L, Mascaro A, Poccia I et al (2012) Lobular breast cancer: same survival and local control compared with ductal cancer, but should both be treated the same way? Analysis of an institutional database over a 10-year period. Ann Surg Oncol 19:1107-1114

2. Guiu S, Wolfer A, Jacot W et al (2014) Invasive lobular breast cancer and its variants: how special are they for systemic therapy decisions? Crit Rev Oncol Hematol 92:235-257

3. Coates AS, Winer EP, Goldhirsch A et al (2015) Tailoring therapies-improving the management of early breast cancer: St Gallen International Expert Consensus on the Primary Therapy of Early Breast Cancer 2015. Ann Oncol 26:1533-1546

4. Straver ME, Rutgers EJ, Rodenhuis S et al (2010) The relevance of breast cancer subtypes in the outcome of neoadjuvant chemotherapy. Ann Surg Oncol 17:2411-2418

5. Petrelli F, Barni S (2013) Response to neoadjuvant chemotherapy in ductal compared to lobular carcinoma of the breast: a metaanalysis of published trials including 1764 lobular breast cancer. Breast Cancer Res Treat 142:227-235

6. Truin W, Vugts G, Roumen RM et al (2016) Differences in response and surgical management with neoadjuvant chemotherapy in invasive lobular versus ductal breast cancer. Ann Surg Oncol 23:51-57

7. Loibl S, Volz C, Mau C et al (2014) Response and prognosis after neoadjuvant chemotherapy in 1051 patients with infiltrating lobular breast carcinoma. Breast Cancer Res Treat 144:153-162

8. Bartlett JM, Brookes CL, Robson T et al (2011) Estrogen receptor and progesterone receptor as predictive biomarkers of response to endocrine therapy: a prospectively powered pathology study in the Tamoxifen and Exemestane Adjuvant Multinational trial. J Clin Oncol 29:1531-1538

9. Van de Water W, Fontein DB, van Nes JG et al (2013) Influence of semi-quantitative oestrogen receptor expression on adjuvant endocrine therapy efficacy in ductal and lobular breast cancer-a TEAM study analysis. Eur J Cancer 49:297-304

10. Truin W, Voogd AC, Vreugdenhil G et al (2011) Influence of histology on the effectiveness of adjuvant chemotherapy in patients with hormone receptor positive invasive breast cancer. The Breast 20:505-509

11. Truin W, Voogd AC, Vreugdenhil G et al (2012) Effect of adjuvant chemotherapy in postmenopausal patients with invasive ductal versus lobular breast cancer. Ann Oncol 23:2859-2865

12. Brouckaert O, Wildiers H, Neven P (2013) Different outcome variables yield different results! Ann Oncol 24:554

13. Katz A, Saad ED, Porter P, Pusztai L (2007) Primary systemic chemotherapy of invasive lobular carcinoma of the breast. Lancet Oncol 8:55-62

14. Purushotham A, Pinder S, Cariati M, Harries M, Goldhirsch A (2010) Neoadjuvant chemotherapy: not the best option in estrogen receptor-positive, HER2-negative, invasive classical lobular carcinoma of the breast? J Clin Oncol 28:3552-3554

15. Rakha EA, El-Sayed ME, Powe DG et al (2008) Invasive lobular carcinoma of the breast: response to hormonal therapy and outcomes. Eur J Cancer 44:73-83

16. Ciriello G, Sinha R, Hoadley KA et al (2013) The molecular diversity of Luminal A breast tumors. Breast Cancer Res Treat 141:409-420

17. Coates AS, Winer EP, Goldhirsch A et al (2015) Tailoring therapies-improving the management of early breast cancer: St Gallen International Expert Consensus on the Primary Therapy of Early Breast Cancer 2015. Ann Oncol 26:1533-1546 